\title{
Fusarium verticillioides GAP1, a gene encoding a putative glycolipid-anchored surface protein, participates in conidiation and cell wall structure but not virulence
}

\author{
Uma Shankar Sagaram, Brian D. Shaw and Won-Bo Shim
}

Correspondence

Won-Bo Shim

wbshim@tamu.edu

Received 1 March 2007

Revised 3 May 2007

Accepted 9 May 2007

\author{
Department of Plant Pathology and Microbiology, Program for the Biology of Filamentous Fungi, \\ Texas A\&M University, College Station, TX 77843-2132, USA
}

\section{INTRODUCTION}

Fusarium verticillioides (Sacc.) Nirenberg (teleomorph: Gibberella moniliformis Wineland) causes rots of maize (Zea mays) stalks and ears (Munkvold \& Desjardins, 1997). Stalk rot of maize is widespread in dry, warm climates (White, 1999), and can cause losses in several different ways, such as stalk breakage, lodging, interruption of the normal grain-filling process, and sometimes premature death of the plant (Dodd, 1980; Michaelson, 1957; White, 1999). On maize ears, the fungus can produce

Abbreviations: CFW, Calcofluor white; ConA, concanavalin A; CR, Congo red; EST, expressed sequence tag; $\mathrm{FB}_{1}$, fumonisin $\mathrm{B}_{1}$; $\mathrm{GAS}$ protein, glycolipid-anchored surface protein; GPI, glycosylphosphatidylinositol; $\mathrm{SSH}$, suppression subtractive hybridization.

The GenBank/EMBL/DDBJ accession no. for the 350 bp GAP1 gene sequence from $F$. verticillioides is DQ458798.

Figures showing the $F$. verticillioides seedling rot assay on hybrid sweetcorn, and Northern analysis of GAP2 and GAP3 expression in F. verticillioides, are available as supplementary data with the online version of this paper. polyketide-derived carcinogenic mycotoxins called fumonisins (Nelson et al., 1993; Marasas, 2001). Since the discovery of fumonisins, several efforts have been made to understand the regulation of fumonisin biosynthesis in the F. verticillioides-maize system (reviewed by Sagaram et al., 2006). Briefly, fumonisins are synthesized by a cluster of 22 FUM genes, of which 15 are co-regulated (Proctor et al., $1999,2003)$. Recently, a putative regulatory gene within the FUM cluster, FUM21, has been identified and characterized (Brown et al., 2007). However, FUM21 gene deletion is not sufficient to completely block fumonisin biosynthesis, suggesting that transcriptional regulation of FUM genes is also affected by regulatory genes outside the cluster. Fumonisin biosynthesis is also impacted by several environmental factors, including available nitrogen, ambient $\mathrm{pH}$ and maize kernel constitution environment (reviewed by Sagaram et al., 2006).

The first regulatory gene identified by the restriction enzyme-mediated integration (REMI) strategy was FCC1, which encodes a C-type cyclin (Shim \& Woloshuk, 2001). Mutation in FCC1 leads to a severe reduction in 
conidiation and fumonisin production. Nitrogen source and $\mathrm{pH}$ strongly influence the conidiation and expression of the fumonisin biosynthetic gene (FUM1) in a $\Delta f c c 1$ strain, indicating a regulatory role for $F C C 1$ in fumonisin biosynthesis and fungal development (Shim \& Woloshuk, 2001). Subsequent genomic approaches, such as construction of cDNA subtraction libraries using a wild-type strain and $\Delta f c c 1$ strains (Shim \& Woloshuk, 2001), and microarray analysis (Pirttilä et al., 2004), have identified additional regulatory genes for fumonisin biosynthesis. As a result, several genes, e.g. FCK1, a C-type cyclindependent kinase, $P A C 1$, a $\mathrm{pH}$ regulatory gene, $Z F R 1$, a $\mathrm{Zn}(\mathrm{II}) 2 \mathrm{Cys} 6$ gene, and $A M Y 1$, a gene encoding a putative $\alpha$-amylase (reviewed by Sagaram et al., 2006), have been identified and characterized.

During the screening of $F$. verticillioides EST index (TIGR) and suppression subtractive hybridization (SSH) cDNA libraries (Shim \& Woloshuk, 2001), a 350 bp EST was identified in the wild-type SSH library that encodes a protein sequence with high homology to glycosylphosphatidylinositol (GPI)-anchored $\beta$-1,3-glucanosyltransferases that belong to a class of GPI-anchored glycoproteins. We designated this gene GAP1 (GPI-anchored protein 1). GPI-anchored cell wall proteins (GPI-CWPs) are among the most common proteins in eukaryotic cell walls (De Groot et al., 2005) that are covalently linked to $\beta-1,6$-glucan, which can be further linked to $\beta$-1,3-glucan or chitin, resulting in a strong anchorage of these proteins to cell walls (Kapteyn et al., 1996, 1997; Kollar et al., 1997). The $\beta$-1,3glucanosyltransferases are involved in the elongation of $\beta$ 1,3-glucan side chains of the cell wall (Mouyna et al., 2000). These enzymes split the $\beta$-1,3-glucan molecule and transfer the newly produced reducing end to the non-reducing end of another $\beta$-1,3-glucan molecule (Mouyna et al., 2000). GPI-anchored $\beta$-1,3-glucanosyltransferases have been shown to play a critical role in fungal cell wall biogenesis (Mouyna et al., 2000), morphogenesis and virulence in opportunistic fungal pathogens of humans (Muhlschlegel \& Fonzi, 1997; DeBernardis et al., 1998; Ghannoum et al., 1995; Mouyna et al., 2005). For example, GEL1 and GEL2 are GPI-anchored $\beta$-1,3-glucanosyltransferase-encoding genes characterized in Aspergillus fumigatus (Mouyna et al., 2000, 2005). Disruption of GEL1 does not result in a phenotype, while disruption of GEL2, and disruption of both GEL1 and GEL2, result in altered cell wall composition, slower growth, unusual conidiogenesis and reduced invasive aspergillosis in a murine model (Mouyna et al., 2005). Significantly, a $\beta$-1,3-glucanosyltransferase-encoding gene, $G A S 1$, is also involved in virulence in the plant-pathogenic fungus Fusarium oxysporum (Caracuel et al., 2005), suggesting that GAP1 may be associated with fungal virulence in F. verticillioides. Caracuel et al. (2005) have also reported that F. oxysporum GAS1 expression is $\mathrm{pH}$ independent, in contrast to Candida albicans PHR1 and PHR2 (Muhlschlegel \& Fonzi, 1997).

The fact that the GAP1 EST was isolated in the F. verticillioides wild-type SSH cDNA library (Shim \&
Woloshuk, 2001) prompted us initially to hypothesize that GAP1 is primarily associated with fumonisin biosynthesis. However, we also argued that GAP1 could be primarily associated with fungal development and conidiation (Mouyna et al., 2000; Shim \& Woloshuk, 2001), which in turn may affect fungal virulence (Caracuel et al., 2005). Thus, we formulated a hypothesis that GAP1 regulates fungal development and virulence in $F$. verticillioides. To test this hypothesis, we generated GAP1 deletion mutants of $F$. verticillioides and investigated growth, conidiation and cell wall biogenesis. In addition, we analysed fungal virulence on maize and fumonisin production. In this study, we demonstrate that GAP1 is required for proper growth and conidiation on solid synthetic media and conidiation in liquid media. Our study revealed that GAP1 influences cell wall structure by organizing the carbohydrate deposition in the cell walls when the fungus is growing on solid synthetic media. We also show that GAP1 is not involved in F. verticillioides virulence on maize, and does not positively regulate fumonisin biosynthesis.

\section{METHODS}

Fungal strains, media and growth conditions. The wild-type $F$. verticillioides strain 7600 (M3125; Fungal Genetics Stock Center) and mutant strains generated for this study were stored in $30 \%(\mathrm{v} / \mathrm{v})$ glycerol at $-80{ }^{\circ} \mathrm{C}$. For genomic DNA extraction, the fungus was grown in YEPD medium ( 3 g yeast extract $1^{-1}, 10$ g peptone $1^{-1}$, and $20 \mathrm{~g}$ dextrose $1^{-1}$ ) or potato dextrose broth (PDB) (BD). For production of initial inoculum [for RNA isolation, growth measurements, spore counts, virulence assays and fumonisin $\mathrm{B}_{1}\left(\mathrm{FB}_{1}\right)$ analysis] the fungal strains were grown on $\mathrm{V} 8$ juice agar plates (200 $\mathrm{ml} \mathrm{V8} \mathrm{juice} 1^{-1}, 3 \mathrm{~g} \mathrm{CaCO}_{3} 1^{-1}, 20 \mathrm{~g} \mathrm{agar}^{-1}$ ) for $7-10$ days. The spores were harvested in sterile water, passed through cheesecloth and quantified using a haemocytometer. For RNA isolation, $10^{6}$ spores were inoculated into BSA liquid (BSAL) medium ( $\mathrm{pH} 3.5,6.0$ and 8.5) $\left(1 \mathrm{~g} \mathrm{NH}_{4} \mathrm{H}_{2} \mathrm{PO}_{4} \mathrm{l}^{-1}, 40 \mathrm{~g}\right.$ sucrose $1^{-1}, 3 \mathrm{~g} \mathrm{KH}_{2} \mathrm{PO}_{4} \mathrm{l}^{-1}, 2 \mathrm{~g}$ $\mathrm{MgSO}_{4} \cdot 7 \mathrm{H}_{2} \mathrm{O} \mathrm{l}^{-1}, 5 \mathrm{~g} \mathrm{NaCl} \mathrm{l}^{-1}$ ) or $10^{7}$ spores were inoculated into defined liquid (DL) medium (initial pH 3.0 and 8.0) $\left(1 \mathrm{~g} \mathrm{NH}_{4} \mathrm{H}_{2} \mathrm{PO}_{4}\right.$ $\mathrm{l}^{-1}, 40 \mathrm{~g}$ sucrose $\mathrm{l}^{-1}, 3 \mathrm{~g} \mathrm{KH}_{2} \mathrm{PO}_{4} \mathrm{l}^{-1}, 2 \mathrm{~g} \mathrm{MgSO}_{4} .7 \mathrm{H}_{2} \mathrm{O} \mathrm{l} \mathrm{l}^{-1}, 5 \mathrm{~g}$ $\left.\mathrm{NaCl} \mathrm{l}^{-1}\right)$ and allowed to grow for 7 days at room temperature $(22-$ $23{ }^{\circ} \mathrm{C}$ ) at 125 r.p.m.

Nucleic acid manipulation. Bacterial plasmid DNA was isolated with the Wizard miniprep DNA purification system (Promega). Fungal genomic DNA was extracted by methods described previously (Shim \& Woloshuk, 2001). Total RNA was extracted with an RNeasy Plant Mini kit (Qiagen) or Trizol reagent (Invitrogen) as per the manufacturers' protocols. Southern and Northern analyses were performed following standard procedures (Sambrook \& Russell, 2001). For Southern analysis, genomic DNA $(10 \mu \mathrm{g})$ was digested with EcoRI and subjected to electrophoresis on a $1 \%$ agarose gel. The DNA was transferred onto a nylon membrane and probed with a ${ }^{32} \mathrm{P}-$ labelled DNA fragment amplified from $F$. verticillioides genomic DNA with primers FvGAS-F4t and FvGAS-R3 (Table 1). For Northern analysis, total RNA was subjected to electrophoresis on a $1.2 \%$ denaturing agarose gel, transferred onto a nylon membrane and hybridized with ${ }^{32} \mathrm{P}$-labelled gene-specific probes. The probes used in all hybridization experiments were ${ }^{32} \mathrm{P}$-labelled with a Prime-It Random Primer Labeling kit (Stratagene).

PCR. All PCR reactions were performed in a GeneAmp PCR system 9700 thermocycler (PE Applied Biosystems). The primers used in this 
Table 1. Primers used in this study

\begin{tabular}{|ll|}
\hline Name & \multicolumn{1}{c|}{ Sequence $\left(\mathbf{5}^{\prime} \mathbf{}^{\prime} \mathbf{3}^{\prime}\right)$} \\
\hline FvGAS-F5 & TTCGAGTTGTCGTCGTCTGAACCA \\
FvGAS-R2t & TCACTGGCCGTCGTTTACAATAAAGCAGTCTAGCTACGGGACCA ${ }^{*}$ \\
FvGAS-F2 & AACAACGGCATAACCAGAAACGCC \\
FvGAS-F4t & CATGGTCATAGCTGTTTCCTGATGCCATCTTGCGCGATTGCTATG ${ }^{\prime}$ \\
FvGAS-R3 & TTGAGCGTGTCTAATGGAGCTGGT \\
FvGAS-R4 & TTCCAAGTCAGGTTCGCAACAACG \\
FvGAS-R5 & ACCAAAGTTCTCGTCATCCTCGCT \\
FvGAS-F7t & CATGGTCATAGCTGTTTCCTGTTCGAGTTGTCGTCGTCTGAACCA $\dagger$ \\
M13 F & TTGTAAAACGACGGCCAGTGA \\
M13-R & CAGGAAACAGCTATGACCATG \\
FvGaseRho-D1 & AAACAGCCTTATCATGCAACGGGC \\
FvGaseRho-U1 & AGAGCTTGATTTGGCGCTTGTTGG \\
FvGAP2-F1 & GATCCCGATGTTTGCAAGCGTGAT \\
FvGAP2-R1 & ATCATCATCATCAGAGGCAGAGGC \\
FvGAP3-F1 & ACACAGCCTACCTTAATCGCACCT \\
FvGAP3-R1 & TGTGTCTGCATCGTTCTTACCGGA \\
\hline
\end{tabular}

*The underlined sequence represents the complementary sequence of the M13-F primer used in double-joint PCR.

$\dagger$ The underlined sequence represents the complementary sequence of the M13-R primer used in double-joint and single-joint PCR.

study are listed in Table 1. PCR amplification of DNA was performed in 25 or $50 \mu$ total volumes with Taq DNA polymerase (Promega) or Expand Long Polymerase (Roche). The PCR conditions were 2 min of pre-denaturation at $94{ }^{\circ} \mathrm{C}$ followed by 30 cycles of $30 \mathrm{~s}$ of denaturation at $94{ }^{\circ} \mathrm{C}, 30 \mathrm{~s}$ of annealing at $55-57^{\circ} \mathrm{C}$, and $1-2 \mathrm{~min}$ of extension at $72{ }^{\circ} \mathrm{C}$ for Taq DNA polymerase and $68{ }^{\circ} \mathrm{C}$ for Expand Long Polymerase.

Deletion and complementation vector construction. Two deletion constructs were generated by the double-joint PCR strategy (Yu et al., 2004) to replace the GAP1 ORF in F. verticillioides strain 7600. First, $1150 \mathrm{bp} 5^{\prime}$ and $1050 \mathrm{bp} 3^{\prime}$ flanking regions of the GAP1 gene were amplified from $F$. verticillioides genomic DNA using Taq DNA polymerase. The primers FvGAS-F2 and FvGAS-R2t were used to amplify the $5^{\prime}$ flanking region, and primers FvGAS-F4t and FvGAS-R4 were used to amplify the $3^{\prime}$ flanking region. Simultaneously, the hygromycin phosphotransferase ( $H Y G)$-encoding gene and the geneticin (G418)-resistance gene (GEN) were amplified from plasmid vectors $\mathrm{pBP} 15$ and $\mathrm{pBS}-\mathrm{G}$, respectively, using the primers M13-F and M13-R. The drug-resistance markers were amplified using Expand Long Polymerase, which has proof-reading activity. Subsequently, the three amplicons were mixed in a single tube in a $1: 3: 1$ ( $5^{\prime}$ fragment: marker: $3^{\prime}$ fragment) molar ratio and joined by PCR without using any primers. Finally, nested primers FvGAS-F5 and FvGAS-R4 were used to amplify the $3.9 \mathrm{~kb}$ amplicon carrying the $H P H$ or GEN marker fused to the GAP1 flanking regions. These fused products, named GAS1DV2-H and GAS1DV2-G, which harbour $H Y G$ and GEN, respectively, were used as GAP1 disruption constructs.

The GAP1 deletion mutant strain GAM126 ( $\triangle g a p 1:: H Y G)$ was complemented with a wild-type GAP1 gene that was fused to GEN. GEN was amplified with primers M13-F and M13-R from pBS-G, and GAP1 [including $1530 \mathrm{bp} 5^{\prime}$ untranslated region (UTR) and $1200 \mathrm{bp}$ 3' UTR] was amplified from genomic DNA with FvGAS-F7t and FvGAS-R3 using Expand Long Polymerase. GEN and GAP1 were fused by a single-joint PCR strategy to generate the complementation construct (Shim et al., 2006; Yu et al., 2004). The joined-PCR product GAS1CV1 was amplified with the primers M13-F and FvGAS-R4 using Expand Long Polymerase and used for transformation to complement GAM126.

Fungal transformation. Fungal protoplasts were prepared and transformed as described previously (Shim \& Woloshuk, 2001), except that mureinase $\left(2 \mathrm{mg} \mathrm{ml}^{-1}\right)$ was replaced by Driselase $(5 \mathrm{mg}$ $\mathrm{ml}^{-1}$ ) (Sigma), and $1 \mathrm{~g}$ wet mycelium was digested in $20 \mathrm{ml}$ enzyme solution [10 $\mathrm{mM} \mathrm{NaH}_{2} \mathrm{PO}_{4}$ (pH 5.8), $20 \mathrm{mM} \mathrm{CaCl}_{2}, \beta$-glucuronidase (1343 $\mathrm{U} \mathrm{ml}^{-1}$; Sigma), Driselase $\left(5 \mathrm{mg} \mathrm{ml}^{-1}\right.$; Sigma), $1.2 \mathrm{M} \mathrm{KCl}$. Transformants were selected on regeneration medium (343 g sucrose $1^{-1}, 0.2 \mathrm{~g}$ yeast extract $1^{-1}, 10 \mathrm{~g}$ agar $\mathrm{l}^{-1}$ ) containing $150 \mu \mathrm{g} \mathrm{ml}$ hygromycin B (Calbiochem) or G-418 (Cellgro).

Mycelial staining, microscopy and cell wall assays. For mycelial staining, $10^{5}$ spores were inoculated on potato dextrose agar (PDA) and allowed to grow. After 2 days, the mycelia were scraped and collected in a $1.5 \mathrm{ml}$ tube. Concanavalin A (ConA) type VI conjugated to FITC (Sigma, catalogue no. C7642) was used at a final concentration of $100 \mu \mathrm{g} \mathrm{ml}^{-1}$ using previously published conditions (Shaw \& Hoch, 1999). ConA in a $100 \mu \mathrm{l}$ volume was added to fungal mycelia and incubated for $45 \mathrm{~min}$. Later, samples were washed twice with $100 \mu \mathrm{l}$ water by successive centrifugation and resuspension. Finally, the samples were resuspended in $100 \mu$ water before observation under the microscope. Microscopic imaging used an Olympus BX51 microscope (Olympus America) fitted with Uplanapo objectives and an Olympus DP70 cooled charge-coupled device (CCD) digital camera. Further details of this imaging system have recently been described (Upadhyay \& Shaw, 2006). Images were acquired using DP70-BSW software (version 01.01) and prepared for publication with Adobe Photoshop. For FITC fluorescence visualization, an Olympus U-MNIBA2 (Olympus) filter cube was used with excitation wavelengths from 470 to $480 \mathrm{~nm}$, emission wavelengths from 510 to $550 \mathrm{~nm}$, and a dichroic mirror at $505 \mathrm{~nm}$ FITC. Susceptibility assays with Congo red (CR) (Matheson Coleman \& Bell) and Calcofluor white (CFW) (fluorescent brightener F3453, 
Sigma) were conducted as described in Ram \& Klis (2006), with few modifications. Ten drops $5 \mathrm{M} \mathrm{KOH}$ was added to dissolve CFW. The assays were conducted on PDA with initial $\mathrm{pH}$ adjusted to 8.0.

Stalk rot and seedling rot assay. Stalk rot assays were performed on 8-week-old plants of the B73 corn line. Internodal regions of the stalk were punctured $\left(2 \mathrm{~mm}\right.$ deep) with a sterile needle and $10^{4}$ spores were inoculated into the punctured hole. Plants were incubated in a growth chamber at $25{ }^{\circ} \mathrm{C}$ and $40 \%$ humidity. Stalks were split open longitudinally after 21 days and the disease symptoms were photographed. For seedling rot assays, the B73 corn line and Golden Cross Bantam hybrid sweetcorn (Ed Hume Seeds) were surface-sterilized with $100 \%$ bleach for $15 \mathrm{~min}$, washed twice with sterile water, and soaked in sterile water for $1 \mathrm{~h}$. The seeds were placed in between two layers of moist filter paper in a sterile glass saucer $(30 \times 15 \mathrm{~cm})$ and allowed to germinate. After 7 days, the top filter paper was removed and the ungerminated seeds were discarded. Seeds were inoculated at the embryo region with $10^{4}$ spores in a $5 \mu \mathrm{l}$ volume. Eight seeds were inoculated for each fungal strain. The glass saucer was covered with Saran wrap and incubated at room temperature $\left(22-23{ }^{\circ} \mathrm{C}\right)$ for 8 days. The experiments were repeated twice with similar results.

$\mathbf{F B}_{\mathbf{1}}$ analysis. $\mathrm{FB}_{1}$ analysis was performed as described previously, with some modifications (Shim \& Woloshuk, 1999). Fungal strains were grown on cracked corn (B73 line; $1 \mathrm{~g}$ dry weight) medium for 15 and 25 days at room temperature $\left(22-23{ }^{\circ} \mathrm{C}\right) . \mathrm{FB}_{1}$ was extracted with acetonitrile: water $(1: 1, \mathrm{v} / \mathrm{v})$ for $48 \mathrm{~h}$. The crude extracts were passed over equilibrated PrepSep SPE C18 columns (Fisher Scientific) to eliminate unwanted metabolites coming from corn and fungus. The $\mathrm{FB}_{1}$ concentration of samples was analysed by HPLC using the Shimadzu LC-20AT HPLC system (Shimadzu Scientific Instruments) equipped with an analytical Zorbax ODS column $(4.6 \times 150 \mathrm{~mm})$ (Agilent Technologies) and a Shimadzu SPD-20A Prominence UV/ Vis detector $(335 \mathrm{~nm})$. The HPLC system was operated following the protocol described by Shim \& Woloshuk (1999). $\mathrm{FB}_{1}$ was quantified by comparing HPLC peak areas with those of $\mathrm{FB}_{1}$ standards (Sigma). Four biological replicates were included in the experiment and the experiment was repeated twice.

F. verticillioides sexual cross experiments Sexual crosses were performed as described in Shim et al. (2006), with modifications. Fresh carrots (400 g) were washed, diced and boiled for $20 \mathrm{~min}$ in $400 \mathrm{ml}$ distilled water. The carrots were macerated and the mixture was passed through cheesecloth. Agar $(20 \mathrm{~g})$ was added to the filtrate and the final volume was brought to 11 with distilled water. The carrot agar medium was autoclaved for $20 \mathrm{~min}$ and poured into $100 \mathrm{~mm}$ diameter Petri plates. Both mating types were grown on V8 agar prior to mating. F. verticillioides 7600, GAM126, GAG8 and GASC1 strains (all with genotype MAT1-1) were crossed with $F$. verticillioides strain 7598 (genotype MAT1-2) (Fungal Genetics Stock Center) on carrot agar plates. The growth conditions were maintained at $25{ }^{\circ} \mathrm{C}$, with a $14 \mathrm{~h}$ light and $10 \mathrm{~h}$ dark cycle.

\section{RESULTS}

\section{Molecular characterization of GAP1}

We isolated a 350 bp EST sequence (wt_0_G15) from the F. verticillioides SSH cDNA library (Shim \& Woloshuk, 2001) that shared high similarity to Saccharomyces cerevisiae GAS protein $\left(E=1 \times 10^{-33}\right)$ and C. albicans $\mathrm{pH}$ responsive protein $\left(E=3 \times 10^{-33}\right)$. The complete GAP1 sequence was obtained from a search of the $F$. verticillioides
7600 strain genome (Broad Institute of Harvard and MIT; http://www.broad.mit.edu). GPI-anchored surface proteins are known to be differentially expressed with changes in ambient pH (Saporito-Irwin et al., 1995; Muhlschlegel \& Fonzi, 1997). In F. verticillioides, GAP1 expression was repressed at $\mathrm{pH} 8.5$ and induced proportionately at acidic pH (Fig. 1). The GAP1 gene is 1669 bp long with a single putative intron (49 bp) and encodes a putative protein of 540 aa. The nucleotide sequence and putative translated protein sequence were submitted to GenBank (accession no. DQ458798). A database search for conserved domains in the Conserved Domain Database (CDD; National Center for Biotechnology Information) revealed the presence of a characteristic glycolipid-anchored surface (GAS) protein (GAS1) domain at the N-terminal region of the protein (Marchler-Bauer et al., 2005). Amino acid comparison using BLASTP analysis indicated that Gap1 shares high homology with predicted protein sequences of other filamentous fungi and yeast (Altschul et al., 1997). Sequence accession numbers of homologues in other fungi with percentage identity to putative Gap1 are: F. oxysporum AAX78216 (94\%); Fusarium graminearum EAA69544 (81\%); Magnaporthe grisea XP_362679 (53\%); Neurospora crassa CAD70754 (51\%); Aspergillus nidulans XP_661334 (50\%) and A. fumigatus EAL84955 (49\%).

\section{Generation of GAP1 deletion mutant}

To test our central hypothesis, we generated $F$. verticillioides GAP1 deletion mutant strains, GAM126 ( $\triangle$ gap 1::HYG) and GAG8 ( $\triangle$ gap 1:: GEN), using a double homologous recombination strategy. After protoplast transformation, hygromycin-resistant colonies were selected and screened for gene deletion by PCR (data not shown). The homologous recombination event was verified by Southern analysis

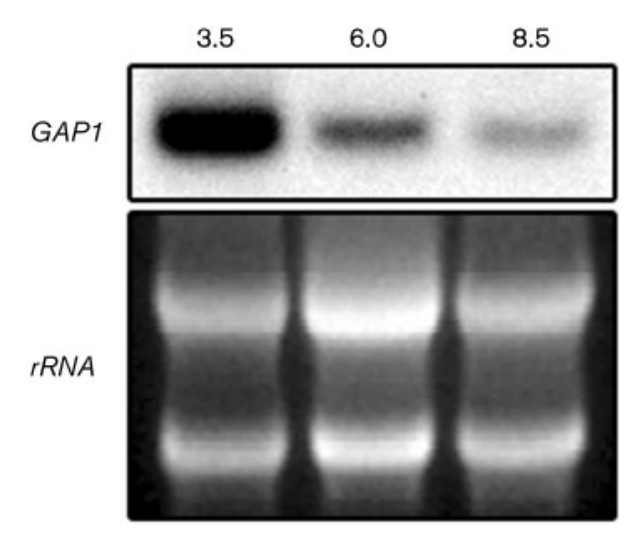

Fig. 1. Northern analysis of GAP1 expression. Total RNA (15 $\mu \mathrm{g})$ was subjected to electrophoresis on a $1.2 \%$ denaturing agarose gel, transferred onto a nylon membrane and hybridized with a ${ }^{32} \mathrm{P}$ labelled probe. A $1 \mathrm{~kb}$ GAP1 genomic DNA fragment amplified with primers FvGAS-F1 and FvGAS-R5 (Table 1) was used as probe. rRNA stained with ethidium bromide is shown as an equal loading control. 
(Fig. 2d). The wild-type strain produced a $5 \mathrm{~kb}$ band, whereas the deletion mutant GAM126 produced a $3 \mathrm{~kb}$ band, indicating that the GAP1 gene was replaced with the disruption construct GAS1DV2-H (Fig. 2b, d). To further validate the GAM126 phenotype, we generated GAG8, a GAP1 knockout strain harbouring a different selectable marker. The deletion of GAP1 in GAG8 was also confirmed by Southern analysis (not shown). Northern analysis showed that the GAP1 expression was completely abolished in both GAM126 and GAG8 strains, whereas the wild-type strain produced a $2.7 \mathrm{~kb}$ transcript (Fig. 3).

\section{Mutation of GAP1 results in cell wall alterations}

Mutational analyses of GPI-anchored proteins in other fungi have resulted in a variety of cell wall development

(a)

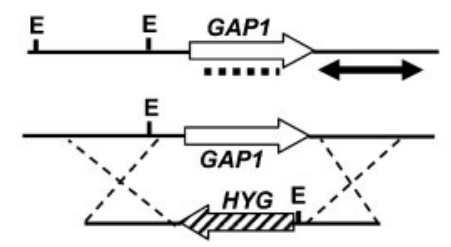

(c)

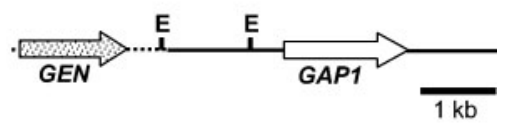

(d)

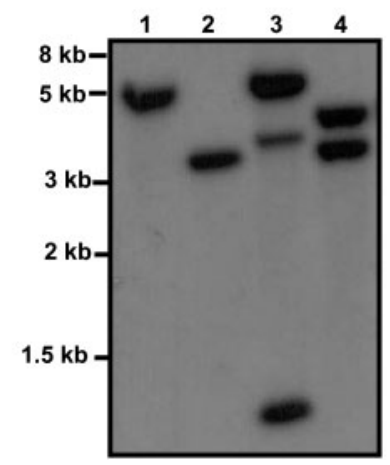

Fig. 2. (a) Schematic representation of a $5.5 \mathrm{~kb} F$. verticillioides GAP1 locus. The dashed line and double arrowhead represent the genomic regions used as probes for Northern and Southern blots, respectively. E, EcoRI restriction site. (b) Schematic representation of targeted replacement of GAP1 with the hygromycin phosphotransferase gene $(H Y G)$ via a double homologous integration event. (c) The complementation construct (GAS1CV1) used to complement GAM126 with a GAP1 wild-type copy. GAS1CV1 was generated by fusing the geneticin-resistance gene (GEN) to the GAP1 gene by single-joint PCR. (d) Southern analysis of transformants. Molecular sizes are indicated on the left. Lanes: 1, wild-type (WT); 2, $\Delta$ gap1:: HYG (GAM126); 3, ectopic integration; 4, complemented strain (GASC1). The wild-type strain produced a $5 \mathrm{~kb}$ band. Homologous recombination of GAP1 with HYG resulted in production of a $3 \mathrm{~kb}$ band due to the insertion of an EcoRl site. The complemented strain produced a mutant band ( $3 \mathrm{~kb}$ ) and a $4 \mathrm{~kb}$ band due to ectopic insertion of the complementation construct.
(a) $\mathrm{pH} 3.0$
(b) $\mathrm{pH} 8.0$

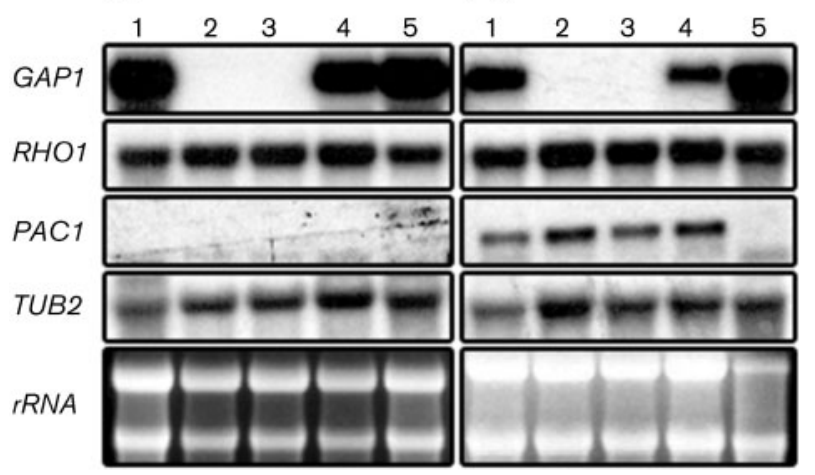

Fig. 3. Northern analysis of gene expression in the wild-type, deletion mutants and complemented strains. Total RNA $(15 \mu \mathrm{g})$ was subjected to electrophoresis on a $1.2 \%$ denaturing agarose gel, transferred onto a nylon membrane and hybridized with appropriate ${ }^{32} \mathrm{P}$-labelled genomic DNA fragments. rRNA stained with ethidium bromide is shown as an equal loading control. Target genes are indicated on the left. Fungal strains: 1, wild-type; 2, GAM126; 3, GAG8; 4, GASC1; 5, PAC2A (PAC1 deletion strain).

defects (Chabane et al., 2006; Davydenko et al., 2005; Kottom et al., 2001; Mouyna et al., 2005), and therefore one of the key biological functions of GAP1 that we were interested in was its involvement in cell wall biogenesis. We noted a brighter CFW staining pattern for the GAP1 deletion strains GAM126 and GAG8 compared to that for the wild-type and the complemented strain GASC1 (data not shown). To better visualize this difference, we assayed the staining pattern of all four strains incubated in ConA conjugated to the fluorophore FITC. ConA is a lectin that selectively binds to glucan and mannan moieties (Shaw \& Hoch, 1999). The GAM126 and GAG8 deletion strains each exhibited a significantly altered and brighter staining pattern than that of the wild-type and GASC1 when observed with identical staining protocols and camera settings (Fig. 4). Deletion strains each exhibited uniformly brightly stained cell walls punctuated by regularly spaced discrete binding foci. The wild-type and the GASC1 strain both lacked significant ConA binding.

Since the cell walls of the deletion strains showed an unusual patterning, we decided to study the expression pattern of RHO1, a gene encoding a small monomeric G protein that is a key regulator of cell wall biogenesis (Bickle et al., 1998; Madaule et al., 1987). RHO1 of F. verticillioides was identified by using the nucleotide sequence of rhol of F. oxysporum (AY884607) as a query in BLASTN analysis of the $F$. verticillioides genome sequence (http://www.broad. mit.edu). The putative translated $\mathrm{RHO} 1$ of $F$. verticillioides is $98 \%$ identical to RHO1 of F. oxysporum. A 500 bp DNA fragment that spans the $R H O 1$ gene was amplified from $F$. verticillioides genomic DNA using primers FvRhoGase-D1 and FvRhoGase-U1, and used as a probe for Northern 

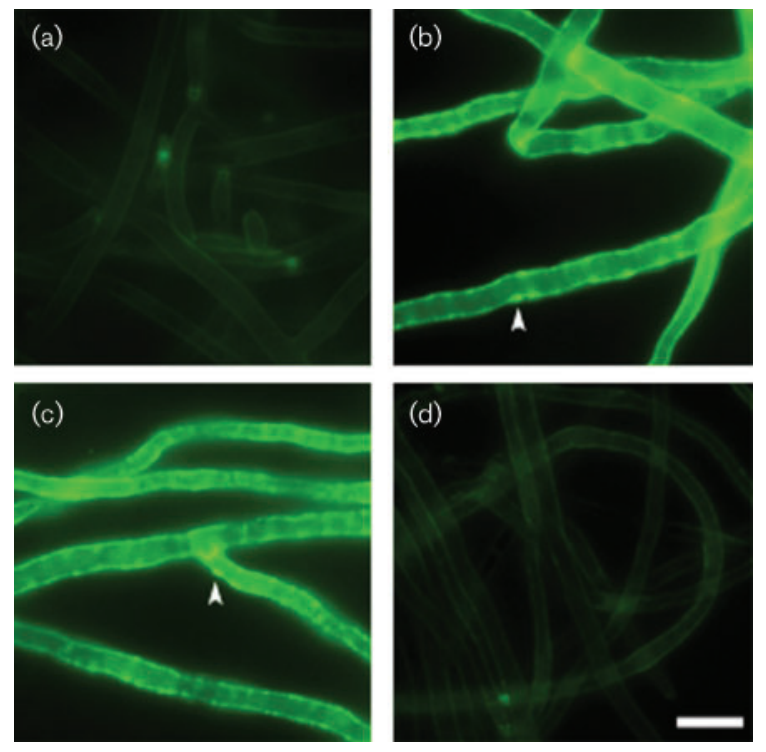

Fig. 4. Staining of fungal cell walls with ConA. Fungal strains: (a) wild-type; (b) GAM126; (c) GAG8; (d) GASC1. Bar, $10 \mu \mathrm{m}$. It should be noted that GAM126 and GAG8 strains exhibited a significantly brighter staining pattern separated by regularly spaced discrete binding foci (arrowheads) compared to that of the wild-type and GASC1. Identical staining protocols, exposure time $(1 / 55 \mathrm{~s})$ and camera settings were used.

analysis. Since GAP1 itself is $\mathrm{pH}$-regulated, we decided to test whether the GAP1-mediated regulation of RHO1 is affected by different $\mathrm{pHs}$. Since $P A C C$-group transcriptional regulators control $\mathrm{pH}$-responsive genes such as PHR1 and PHR2 in C. albicans (Muhlschlegel \& Fonzi, 1997; Porta et al., 1999; Saporito-Irwin et al., 1995), we included an F. verticillioides PAC1-deletion strain to test if PAC1 has a direct effect on GAP1 and/or RHO1. Surprisingly, RHO1 transcript levels in GAM126 and GAG8 mutants were similar to those of the wild-type under both $\mathrm{pH}$ conditions tested (Fig. 3). Our results also indicated that GAP1 expression is induced under alkaline conditions in a PAC1-deletion mutant, whereas RHO1 expression is unaffected (Fig. 3). These results suggest that the deletion of GAP1 in F. verticillioides might alter the composition of the cell wall through a mechanism that does not involve RHO1, unlike in F. oxysporum (Caracuel et al., 2005). Alternatively, it can be hypothesized that the involvement of Rhol in cell wall biogenesis in $F$. verticillioides is post-transcriptional.

We also examined the effect of cell wall stressors such as CR and CFW on the susceptibility of GAM126 and GAG8 (Ram \& Klis, 2006). Surprisingly, the deletion strains were resistant to these compounds compared to their wild-type progenitor. The wild-type strain produced significantly less radial growth on plates containing CR (25 and $50 \mu \mathrm{g} \mathrm{ml}^{-1}$ ) in comparison to a negative control (Fig. 5). In contrast, there was no noticeable difference in the growth of GAM126 and GAG8 in the presence of CR (Fig. 5). The resistant phenotype was not obvious in the first few days after inoculation, but was clearly expressed when observed 5 and 7 days after inoculation (not shown). The complemented strain GASC1 showed a phenotype similar to that of the wild-type. Similar results were observed with CFW at $25 \mu \mathrm{g} \mathrm{ml}^{-1}$ (not shown). At CFW concentrations of $50 \mu \mathrm{g} \mathrm{ml}^{-1}$ or higher, all the strains were highly susceptible, and as a result no growth was observed (not shown).

\section{Deletion of GAP1 affects growth and microconidia production}

Deletion of GAP1 affected growth on synthetic solid media and in suspension in liquid medium (Table 2, Fig. 6). When grown on agar medium, the growth of both the GAM126 strain and the GAG8 strain was restricted when compared to that of the wild-type. The deletion mutants produced a compact mass of mycelium with restricted edges that tended to grow into the agar medium, while their wild-type progenitor produced thin and spreading
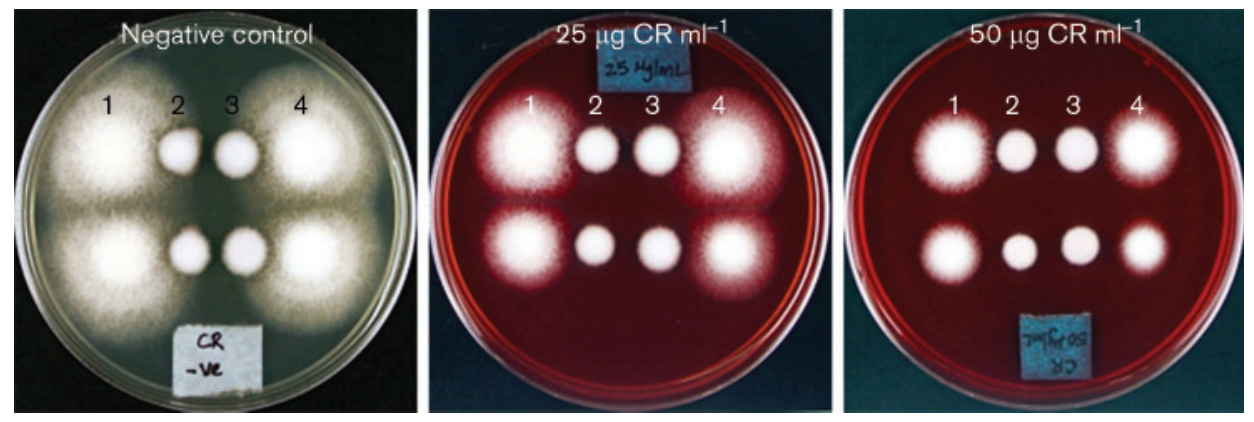

Fig. 5. Effect of $\mathrm{CR}$ on F. verticillioides wild-type and mutant strains. Spore suspension $(2 \mu \mathrm{l})$ containing $2 \times 10^{4}$ (upper rows) and $2 \times 10^{3}$ (lower rows) spores was spot-inoculated on PDA plates with or without CR, and the plates were incubated in the dark at $25{ }^{\circ} \mathrm{C}$. The plates shown here were photographed 5 days after inoculation. Fungal strains: 1, wild-type; 2, GAM126; 3 , GAG8; 4, GASC1. 
Table 2. Comparison of growth and microconidial production between the wild-type and GAP1 deletion strains

Data were analysed using analysis of variance (ANOVA), and significantly different means were separated by least significant difference (LSD). Significantly different means for microconidial number (in the same column) are represented with a, b and $\mathrm{c}(P<0.05)$. All experiments were repeated twice with similar results.

\begin{tabular}{|c|c|c|c|c|c|c|}
\hline \multirow[t]{2}{*}{ Strain ${ }^{\star}$} & \multicolumn{3}{|c|}{ Growth $\dagger$} & \multicolumn{3}{|c|}{ Microconidia $\ddagger$} \\
\hline & $0.5 \times$ PDA $(\mathrm{mm})$ & V8 agar $(\mathrm{mm})$ & $0.5 \times$ PDB $(\mathrm{mg})$ & $0.5 \times \mathrm{PDA} \$, 10^{5}$ & V8 agar $\$, 10^{6}$ & $0.5 \times \mathrm{PDB}, 10^{4}$ \\
\hline Wild-type & $52 \pm 1$ & $66 \pm 1$ & $58 \pm 1$ & $131 \pm 18 \mathrm{a}$ & $70 \pm 7 a$ & $112 \pm 7 \mathrm{a}$ \\
\hline GAM126 & $11 \pm 1$ & $20 \pm 1$ & $58 \pm 1$ & $33 \pm 2 b$ & $12 \pm 1 \mathrm{c}$ & $53 \pm 6 b$ \\
\hline GAG8 & $11 \pm 1$ & $19 \pm 1$ & $60 \pm 1$ & $32 \pm 6 b$ & $14 \pm 1 \mathrm{c}$ & $47 \pm 3 b$ \\
\hline GASC1 & $48 \pm 1$ & $60 \pm 0$ & $58 \pm 1$ & $146 \pm 14 a$ & $57 \pm 12 b$ & $109 \pm 5 \mathrm{a}$ \\
\hline
\end{tabular}

${ }^{\star}$ Fungal strains were spot-inoculated with $10^{4}$ spores (agar plate) or $10^{6}$ spores (in $100 \mathrm{ml}$ stagnant broth) and allowed to grow for 7 days at room temperature $\left(22^{\circ} \mathrm{C}\right)$.

$\dagger$ Growth was measured as diameter $(\mathrm{mm})$ for agar plates and dry mass $(\mathrm{mg})$ for broth. Values represent the mean \pm SD of three biological replicates. $\ddagger$ Mean \pm SD of number of conidia per millilitre.

\$Agar plates were flooded with $10 \mathrm{ml} 0.1 \%$ Triton, passed through eight layers of cheesecloth (VWR) to eliminate mycelium, and microconidia were counted using a haemocytometer.

borders on the surface of the medium (Fig. 6a). Microscopic examination of spore germination revealed that deletion strains produced short and highly branched hyphae, whereas the wild-type strain produced long and less-branched hyphae that extended on the surface of agar
(Fig. 6b). However, we observed no defect in deletion strains with regard to mycelial mass production (Table 2) or filamentous growth (Fig. 6c) when grown in liquid media under shaking conditions. In YEPD suspension, when compared to their wild-type progenitor, GAM126 (a)

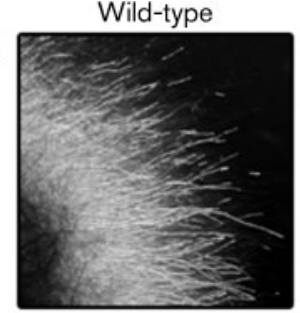

(b)

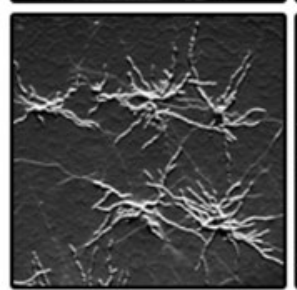

(c)

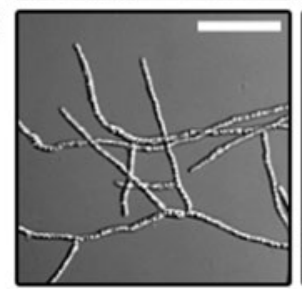

GAM126
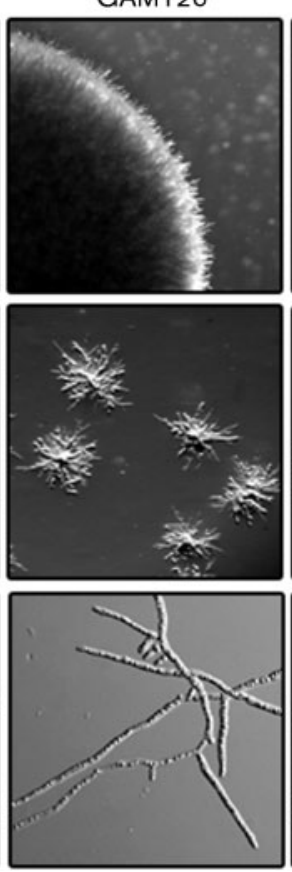

GAG8
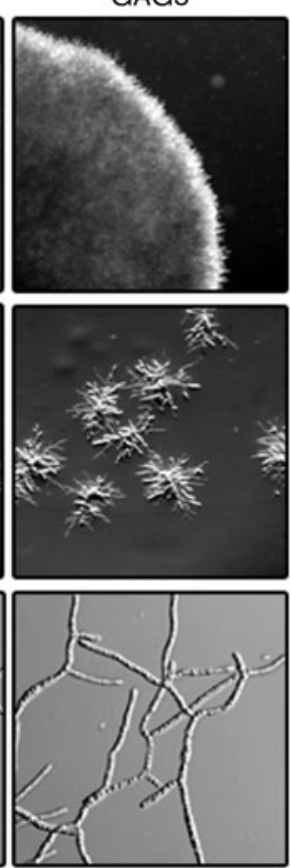

GASC1
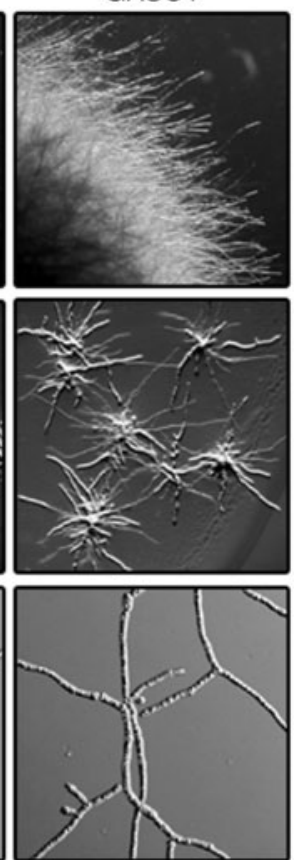

Fig. 6. Effect of GAP1 mutation on growth and hyphal development. (a) Colony edges of fungal strains grown on $0.5 \times$ PDA. (b) Spores $\left(10^{3}\right)$ in a $10 \mu \mathrm{l}$ volume were spot-inoculated on $0.5 \times$ PDA. Pictures of individual colonies were taken 4 days after inoculation. (c) Growth of GAP1 deletion mutants and complemented strains in 0.5 PDB in comparison with that of the wildtype strain. In (a) and (b) it should be noted that the deletion strain produced short and hyper-branching hyphae that resulted in compact and restricted growth on solid media. (a, b) $\times 20$ magnification; (c) $\times 10$ magnification. Bar, $50 \mu \mathrm{m}$. 
and GAG8 produced thin and densely branched hyphae that tended to remain submerged (not shown). In PDB suspension, GAM126 and GAG8 produced densely branched hyphae, but hyphal diameter was not noticeably different from that of the wild-type (not shown).

GAP1 deletion strains produced significantly $(P>0.05)$ fewer microconidia than the wild-type when grown on $0.5 \times$ PDA and V8 agar (Table 2). We speculated that the production of fewer conidia on agar medium might be due to the restricted growth of deletion strains. Hence, we tested the microconidia production in a liquid medium (PDB). Under this condition, GAM126 and GAG8 produced approximately half the microconidia produced by their wild-type progenitor (Table 2).

\section{Deletion of GAP1 did not influence pathogenicity and $F B_{1}$ production}

Previous reports showing that GAS proteins in filamentous fungi are involved in virulence (Caracuel et al., 2005; Martinez-Lopez et al., 2004; Mouyna et al., 2005) prompted us to examine the involvement of GAP1 in F. verticillioides virulence. In the maize stalk rot assay, we tested the ability of deletion strains to infect and invade stalk tissue. As observed 21 days after infection, the deletion strains successfully colonized and rotted the tissue in a similar manner to the wild-type (Fig. 7a). In the maize seedling assay, the deletion strains were as efficient as the wild-type in infecting and colonizing seedlings (Fig. 7b). After 8 days, all the strains colonized the kernels completely, and invaded the radicle and plumule regions of the seedlings. Similar results were observed in both the maize lines tested (Fig. 7b, Supplementary Fig. S1). Based on these results, we concluded that GAP1 is not involved in virulence in $F$. verticillioides.
To determine the effect of the GAP1 mutation on $\mathrm{FB}_{1}$ biosynthesis, we compared the $\mathrm{FB}_{1}$ production ability of GAP1 deletion strains with that of the wild-type strain. Observations made at 25 days post-inoculation revealed that GAM126 and GAG8 strains grew similarly, but TLC analysis showed higher $\mathrm{FB}_{1}$ levels than those of the wildtype strain (not shown). However, further HPLC analysis indicated that only the GAG8 strain produced significantly $(P>0.05)$ higher levels of $\mathrm{FB}_{1}(47.63 \pm 2.87$ p.p.m.) than its wild-type progenitor $\left(29.69 \pm 6.87\right.$ p.p.m.). The $\mathrm{FB}_{1}$ levels produced by GAM126 ( $37.38 \pm 4.79$ p.p.m.) and GASC1 $(26.25 \pm 6.36$ p.p.m. $)$ were not significantly different from those the wild-type strain. Contradictory to our hypothesis, the data clearly indicate that GAP1 is not a positive regulator of $\mathrm{FB}_{1}$ biosynthesis in $F$. verticillioides.

\section{Gene complementation}

To reconfirm that restricted growth and the reduced conidiation phenotype in GAM126 and GAG8 strains is due to the deletion of GAP1, we complemented GAM126 with a wild-type copy of GAP1. Geneticin-resistant transformants were analysed for a functional GAP1 gene by PCR (data not shown) and Southern analysis (Fig. 2c, d). The complemented strain GASC1 contains a $4 \mathrm{~kb}$ fragment, indicative of complementation construct insertion at an ectopic location, in addition to a $3 \mathrm{~kb}$ fragment resulting from GAS1DV2-H. While we observed that the restoration of GAP1 expression in GASC1 was not to that of the wild-type (lane 4, Fig. 3a and b), all the phenotypes observed in GAM126 and GAG8 strains, such as conidiation, growth and cell wall phenotypes, were completely restored to wild-type levels (Figs 4, 5 and 6), providing evidence that GAP1 deletion was the direct cause of GAM126 and GAG8 mutant phenotypes. (a)

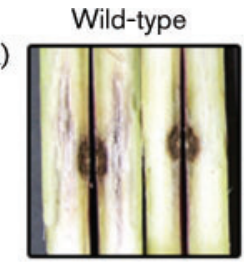

(b)

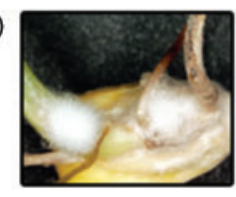

GAM126
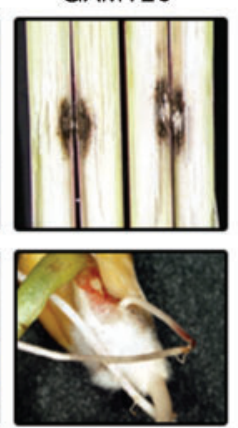

GAG8
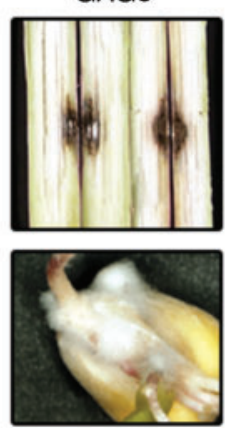

GASC1
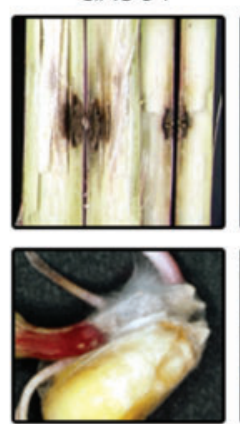
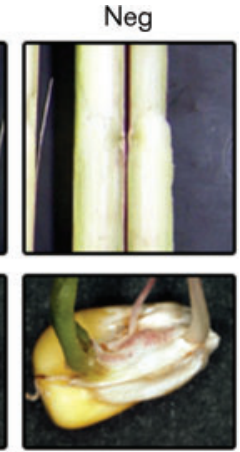

Fig. 7. F. verticillioides stalk rot and seedling rot assay. (a) Eight-week-old maize stalks were inoculated with $10^{4}$ spores of fungal strains at the internodal region and incubated in a growth chamber for 21 days at $25^{\circ} \mathrm{C}$. Subsequently, the maize stalks were split longitudinally to assay the severity of stalk rot. Four independent biological repetitions were performed, and two biological repetitions were selected and are shown in the figure. Maize stalk inoculated with sterile water is shown as the negative (Neg) control. (b) Germinated corn seedlings were inoculated with $10^{4}$ spores of fungal strains at the embryo region. Eight seedlings were infected with each strain. Seedlings were periodically observed and were photographed 8 days after infection. A close-up picture of a single seedling for each strain, showing the fungal colonization, is shown. 


\section{Effect of GAP1 deletion on F. verticillioides sexual development}

We demonstrated that the GAP1 deletion had no impact on sexual development in $F$. verticillioides. Wild-type, GAM126, GAG8 and GASC1 strains were crossed to the opposite mating type wild-type strain 7598 , and after 10 days' incubation at $25{ }^{\circ} \mathrm{C}$ with a $14 \mathrm{~h}$ light and $10 \mathrm{~h}$ dark cycle we observed the development of perithecia as black spots embedded in the mycelium (not shown). Later, we observed viable ascospore formation in all the crosses performed (not shown).

\section{DISCUSSION}

We regard $F C C 1$ as a key regulatory gene that impacts two important biological features, fumonisin biosynthesis and conidiation, in F. verticillioides (Shim \& Woloshuk, 2001). The $F$. verticillioides GAP1 gene was isolated from the wildtype SSH cDNA library, suggesting that GAP1 is downstream of $F C C 1$ in the signalling pathway that regulates fumonisin biosynthesis or conidiation, or both. In silico analysis showed that GAP1 encodes a putative protein that shares high structural similarity to a family of GPIanchored glycoproteins that are known to be involved in cell development in filamentous fungi (Chabane et al., 2006; Mouyna et al., 2000, 2005). Therefore, we hypothesized that GAP1 is primarily associated with conidiation and fungal development. While earlier studies have shown that osmotic stabilization with $1.2 \mathrm{M}$ sorbitol partially remediates the restricted colony phenotype in a gas 1 mutant (Caracuel et al., 2005), the restricted colony growth phenotypes in GAM126 and GAG8 were not remediated upon addition of osmotic stabilizers, including $1.2 \mathrm{M} \mathrm{KCl}$ and $1.2 \mathrm{M}$ sorbitol (not shown).

When mycelia of wild-type, GAM126 and GAG8 were stained with CFW, we noticed that the cell walls of the GAM126 and GAG8 strains were more brightly stained than those of the wild-type, indicating either that the mutant strains produced more chitin and $\beta$-glucans in their cell walls, or that these moieties were more readily stained by CFW in the mutant (not shown). When stained with ConA-FITC, GAM126 and GAG8 exhibited uniform binding of the lectin to cell walls, punctuated by discrete sites of greater binding (Fig. 4). In contrast, neither the wild-type nor GASC1 revealed any significant ConA binding sites. ConA is routinely used to assay the presence of glucan and mannan moieties in fungal cell walls (Shaw \& Hoch, 1999), and is generally believed to bind to terminal $\alpha$-mannan and $\alpha$-glucan (Smith \& Goldstein, 1967). One might have hypothesized that the GAP1 mutants would exhibit fewer ConA binding sites than the wild-type, since the GAP1 protein is thought to function in the linking of $\beta$ linked glucan groups. Our result is not surprising, however, since the alteration of the cell wall by lack of GAP1 function may lead to a more amorphous cell wall that is less crosslinked, therefore leading to exposure of more ConA binding sites. While it is difficult to unambiguously explain this ConA staining pattern, it is clear from our results that the GAP1 mutant cell wall is significantly altered from that of the wild-type. In this study, we also observed that GAM126 and GAG8 strains were resistant to the cell wallstressing agents CR and CFW. This result contrasts with those obtained with $S$. cerevisiae gas1 mutants, which are sensitive to CFW (Popolo et al., 1993). Interestingly, resistance to CR and CFW has also been observed in $S$. cerevisiae mutants that are defective in chitin synthesis (Imai et al., 2005; Roncero et al., 1988). In general, the cell wall mutants that are disrupted in the synthesis of $\beta-1,3$ or $\beta-1,6$ glucans, GPI-anchored glycoprotein biosynthesis or mannosylation of mannoproteins have elevated chitin levels and are hypersensitive to CR and CFW (Imai et al., 2005; Ram et al., 1994). However, in F. verticillioides, we hypothesize that deletion of GAP1 results in reduced chitin production that renders the mutants resistant to CR and CFW.

The expression of $\mathrm{pH}$-responsive genes in filamentous fungi is regulated by key transcriptional regulators such as pacC in A. nidulans (Tilburn et al., 1995) and PRR1 in C. albicans (Porta et al., 1999). For example, the expression of PHR1 and PHR2, the $\beta$-1,3-glucanotransferase-encoding genes in $C$. albicans, is regulated by changes in the external pH via PRR1 (Muhlschlegel \& Fonzi, 1997; Porta et al., 1999; Saporito-Irwin et al., 1995). In contrast, GEL1 and GEL2, the two characterized $\beta$-1,3-glucanotransferaseencoding genes in A. fumigatus, are constitutively expressed over a range of growth conditions (Mouyna et al., 2000). Interestingly, the expression of GAS1 in F. oxysporum is not controlled by ambient $\mathrm{pH}$, or by the responsive regulator PacC (Caracuel et al., 2005). In contrast to the results of $F$. oxysporum studies, the expression of GAP1 in F. verticillioides is induced under acidic conditions (Fig. 1) and seems to be dependent upon the $\mathrm{pH}$-responsive transcription factor PAC1 (Fig. 3). Our Northern analysis revealed that GAP1 expression is upregulated under alkaline conditions in a PAC1 deletion mutant compared to that of the wild-type (Fig. 3b, lane 5). We propose that under alkaline conditions, induction of PAC1 suppresses the expression of GAP1 and vice versa. However, further studies are needed to confirm the regulation of GAP1 by $P A C 1$. Since ambient $\mathrm{pH}$ plays a critical role in the expression of GAP1, and GAP1 plays an important role in growth, we decided to check if the $\mathrm{pH}$ variation affects the growth of GAP1 deletion strains. Unlike the C. albicans deletion strains (Muhlschlegel \& Fonzi, 1997; SaporitoIrwin et al., 1995), differences in $\mathrm{pH}$ (4.5 and 7.5) did not affect the growth of the GAM126 and GAG8 strains in either solid or liquid media (not shown).

In addition to investigating the developmental phenotypes of GAM126 and GAG8, we studied the impact of $\Delta$ gap 1 on key biological features that are linked to the FCC1 in F. verticillioides. In our study we observed a decrease in production of conidia due to the deletion of GAP1 (Table 2). Flaherty et al. (2003) have shown that PAC1 is 
critical to maintain proper growth and conidiation under alkaline conditions. However, the ambient $\mathrm{pH}$ did not have any impact on growth in the GAM126 and GAG8 strains. Hence, we speculate that the reduction in microconidia production in our deletion mutants was due to a developmental defect similar to the abnormal conidiophores observed in A. fumigatus $\Delta$ gel2 and $\Delta$ gel2 $\Delta$ gel1 mutants (Mouyna et al., 2005), rather than molecular regulation of conidiation genes. Overall, very little information is available regarding GPI-anchored proteins in relation to conidiation. Identification and characterization of developmental-related regulatory genes in $F$. verticillioides are necessary to clarify this relationship. We were intrigued that GAP1 deletion did not have a negative impact on fumonisin production, which was in contrast to the conidiation data. Rather, we observed an increased level of $\mathrm{FB}_{1}$ production in the GAG8 strain. These data provide indirect evidence that the primary functional role of GAP1 is to regulate proper hyphal development and conidiation in F. verticillioides. The discovery of additional genes in the FCC1-mediated signalling pathways will allow us to investigate the epistatic relationship between the genes and clarify the pathway-phenotype association.

In this report we also demonstrated that GAP1 in F. verticillioides is not essential for invasion and colonization of maize stalks and seedlings. In a way it is interesting that the GAM126 and GAG8 strains, which are severely impaired in hyphal development on synthetic solid surfaces, can grow as effectively as the wild-type in maize stalks and on seedlings that in theory can be considered to be solid surfaces. Based on our observations it is unlikely that GAP1 plays a signalling role in the interaction between maize and $F$. verticillioides. Our finding is significant, based on the fact that supposedly orthologous proteins ( $94 \%$ identical) performed dissimilar functions (Caracuel et al., 2005; this study), although both fungi might have different modes of infection. However, our finding is not surprising, since the fungus might have a compensatory mechanism to overcome the loss of GAP1. Similar results have been observed in other filamentous fungi. For example, deletion of GEL1 in A. fumigatus does not have an impact on virulence, but deletion of its homologue GEL2 affects morphogenesis and virulence (Mouyna et al., 2005). Surprisingly, GEL1 and GEL2 of A. fumigatus both complement a GAS1 deletion strain of $S$. cerevisiae, indicating that both proteins can act as functional homologues in yeast (Mouyna et al., 2005). In addition, under in vitro conditions, Gel2p exhibits the same enzymic activity as Gellp, reconfirming the redundancy in function (Mouyna et al., 2005). The regulation of expression of structurally similar but functionally different genes could be under the control of external signals such as $\mathrm{pH}$, nutrient source, etc. On the other hand, PHR1 and PHR2 are GPIanchored proteins in $C$. albicans that structurally and functionally exhibit opposing relationships between $\mathrm{pH}$ and morphology: PHR1 and PHR2 mutants are morphologically abnormal at alkaline and acidic $\mathrm{pH}$, respectively (Muhlschlegel \& Fonzi, 1997; Saporito-Irwin et al., 1995).
The fact that deletion of GAP1 had no effect on pathogenicity, whereas its structural homologue in $F$. oxysporum has a significant impact on virulence (Caracuel et al., 2005), further indicates that the function of GPI proteins is specific to each organism.

Analyses of publicly available fungal genomes revealed that more than one GAS protein family gene member is present in filamentous fungi, and as expected two additional GPIanchored glycoprotein-encoding genes were identified in the $F$. verticillioides genome. This might indicate that $\beta-1,3-$ glucanotransferases are functionally homologous or that they perform a different function. To address this question, we first studied the expression of the other two GAP protein-encoding genes in $F$. verticillioides. The complete GAP2 (supercontig 3; locus FVEG_02799.3) and GAP3 (supercontig 5; locus FVEG_04666.3) sequences were obtained from a genome search of the $F$. verticillioides 7600 strain (Broad Institute of Harvard and MIT; http:// www.broad.mit.edu). Our Northern analysis indicated that GAP2 expression is also dependent on ambient $\mathrm{pH}$ (Supplementary Fig. S2). In contrast to GAP1 expression, GAP2 is induced under alkaline conditions (Supplementary Fig. S2). No detectable GAP3 transcript levels were observed under the conditions tested (Supplementary Fig. S2). Our studies also revealed that GAP2 expression is unaltered in the GAM126 and GAM8 strains under both $\mathrm{pH}$ (3.0 and 8.0) conditions tested (not shown). This suggests that GAS proteins (at least Gap1 and Gap2) in F. verticillioides are regulated by ambient $\mathrm{pH}$, probably through $\mathrm{pH}$ transcription factor Pac1, and might perform their functions independently of one other. Further mutational studies are needed to better understand the role of GAP2 and GAP3 proteins in F. verticillioides.

Finally, it is evident that $\beta$-1,3-glucanotransferases share a common function of involvement in cell wall biogenesis. In spite of their homology, GPI proteins of fungi are regulated in different ways and perform different cellular functions, probably to meet the specific requirements of an organism.

\section{ACKNOWLEDGEMENTS}

Financial support was provided by the US Department of Agriculture National Research Initiative (USDA-NRI) Competitive Grants Program, award no. 05-35201-16233.

\section{REFERENCES}

Altschul, S. F., Madden, T. L., Schaffer, A. A., Zhang, J., Zhang, Z., Miller, W. \& Lipman, D. J. (1997). Gapped BLAST and PSI-BLAST: a new generation of protein database search programs. Nucleic Acids Res 25, 3389-3402.

Bickle, M., Delley, P. A., Schmidt, A. \& Hall, M. N. (1998). Cell wall integrity modulates RHO1 activity via the exchange factor ROM2. EMBO J 17, 2235-2245.

Brown, D. W., Butchko, R. A., Busman, M. \& Proctor, R. (2007). The Fusarium verticillioides FUM gene cluster encodes a Zn(II)2Cys6 
protein that affects FUM gene expression and fumonisin production. Eukaryot Cell (in press). http://dx.doi.org/10.1128/EC.00400-06

Caracuel, Z., Martinez-Rocha, A. L., Di Pietro, A., Madrid, M. P. \& Roncero, M. I. (2005). Fusarium oxysporum gas 1 encodes a putative beta-1,3-glucanosyltransferase required for virulence on tomato plants. Mol Plant Microbe Interact 18, 1140-1147.

Chabane, S., Sarfati, J., Ibrahim-Granet, O., Du, C., Schmidt, C., Mouyna, I., Prevost, M. C., Calderone, R. \& Latge, J. P. (2006). Glycosylphosphatidylinositol-anchored Ecm33p influences conidial cell wall biosynthesis in Aspergillus fumigatus. Appl Environ Microbiol 72, 3259-3267.

Davydenko, S. G., Feng, D., Jantti, J. \& Keranen, S. (2005). Characterization of GPI14/YJR013w mutation that induces the cell wall integrity signalling pathway and results in increased protein production in Saccharomyces cerevisiae. Yeast 22, 993-1009.

De Bernardis, F., Muhlschlegel, F. A., Cassone, A. \& Fonzi, W. A. (1998). The $\mathrm{pH}$ of the host niche controls gene expression in and virulence of Candida albicans. Infect Immun 66, 3317-3325.

De Groot, P. W. J., Ram, A. F. \& Klis, F. M. (2005). Features and functions of covalently linked proteins in fungal cell walls. Fungal Genet Biol 42, 657-675.

Dodd, J. L. (1980). The role of plant stresses in development of corn stalk rots. Plant Dis 64, 533-537.

Flaherty, J. E., Pirttila, A. M., Bluhm, B. H. \& Woloshuk, C. P. (2003). $P A C 1$, a pH-regulatory gene from Fusarium verticillioides. Appl Environ Microbiol 69, 5222-5227.

Ghannoum, M. A., Spellberg, B., Saporito-Irwin, S. M. \& Fonzi, W. A. (1995). Reduced virulence of Candida albicans PHR1 mutants. Infect Immun 63, 4528-4530.

Imai, K., Noda, Y., Adachi, H. \& Yoda, K. (2005). A novel endoplasmic reticulum membrane protein Rcrl regulates chitin deposition in the cell wall of Saccharomyces cerevisiae. J Biol Chem 280, 8275-8284.

Kapteyn, J. C., Montijn, R. C., Vink, E., De la Cruz, J., Llobell, A., Douwes, J. E., Shimoi, H., Lipke, P. N. \& Klis, F. M. (1996). Retention of Saccharomyces cerevisiae cell wall proteins through a phosphodiester-linked $\beta$-1,3-/ $\beta$-1,6-glucan heteropolymer. Glycobiology $\mathbf{6}$, 337-345.

Kapteyn, J. C., Ram, A. F., Groos, E. M., Kollar, R., Montijn, R. C., Van den Ende, H., Llobell, A., Cabib, E. \& Klis, F. M. (1997). Altered extent of cross-linking of $\beta 1,6$-glucosylated mannoproteins to chitin in Saccharomyces cerevisiae mutants with reduced cell wall $\beta 1,3$-glucan content. J Bacteriol 179, 6279-6284.

Kollar, R., Reinhold, B. B., Petrakova, E., Yeh, H. J., Ashwell, G., Drgonova, J., Kapteyn, J. C., Klis, F. M. \& Cabib, E. (1997). Architecture of the yeast cell wall. $\beta(1 \rightarrow 6)$-glucan interconnects mannoprotein, $\beta(1 \rightarrow 3)$-glucan, and chitin. J Biol Chem 272, 17762-17775.

Kottom, T. J., Thomas, C. F., Jr \& Limper, A. H. (2001). Characterization of Pneumocystis carinii PHR1, a pH-regulated gene important for cell wall integrity. J Bacteriol 183, 6740-6745.

Madaule, P., Axel, R. \& Myers, A. M. (1987). Characterization of two members of the rho gene family from the yeast Saccharomyces cerevisiae. Proc Natl Acad Sci U S A 84, 779-783.

Marasas, W. F. O. (2001). Discovery and occurrence of the fumonisins: a historical perspective. Environ Health Perspect 109, 239-243.

Marchler-Bauer, A., Anderson, J. B., Cherukuri, P. F., DeWeeseScott, C., Geer, L. Y., Gwadz, M., He, S., Hurwitz, D. I., Jackson, J. D. \& other authors (2005). CDD: a Conserved Domain Database for protein classification. Nucleic Acids Res 33, D192-D196.

Martinez-Lopez, R., Monteoliva, L., Ez-Orejas, R., Nombela, C. \& Gil, C. (2004). The GPI-anchored protein CaEcm 33 p is required for cell wall integrity, morphogenesis and virulence in Candida albicans. Microbiology 150, 3341-3354.

Michaelson, M. E. (1957). Factors affecting development of stalk rots of corn caused by Diplodia zeae and Gibberella zeae. Phytopathology 47, 499-503.

Mouyna, I., Fontaine, T., Vai, M., Monod, M., Fonzi, W. A., Diaquin, M., Popolo, L., Hartland, R. P. \& Latge, J. P. (2000). Glycosylphosphatidylinositol-anchored glucanosyltransferases play an active role in the biosynthesis of the fungal cell wall. J Biol Chem 275, 14882-14889.

Mouyna, I., Morelle, W., Vai, M., Monod, M., Lechenne, B., Fontaine, T., Beauvais, A., Sarfati, J., Prevost, M. C. \& other authors (2005). Deletion of GEL2 encoding for a beta(1-3)glucanosyltransferase affects morphogenesis and virulence in Aspergillus fumigatus. Mol Microbiol 56, 1675-1688.

Muhlschlegel, F. A. \& Fonzi, W. A. (1997). PHR2 of Candida albicans encodes a functional homolog of the $\mathrm{pH}$-regulated gene PHR1 with an inverted pattern of pH-dependent expression. Mol Cell Biol 17, 5960-5967.

Munkvold, G. P. \& Desjardins, A. E. (1997). Fumonisins in maize: can we reduce their occurrence? Plant Dis 81, 556-565.

Nelson, P. E., Desjardins, A. E. \& Plattner, R. D. (1993). Fumonisins: mycotoxins produced by fusarium species: biology, chemistry, and significance. Annu Rev Phytopathol 31, 233-252.

Pirttilä, A. M., Mclntyre, L. M., Payne, G. A. \& Woloshuk, C. P. (2004). Expression profile analysis of wild-type and $f c c 1$ mutant strains of Fusarium verticillioides during fumonisin biosynthesis. Fungal Genet Biol 41, 647-656.

Popolo, L., Vai, M., Gatti, E., Porello, S., Bonfante, P., Balestrini, R. \& Alberghina, L. (1993). Physiological analysis of mutants indicates involvement of the Saccharomyces cerevisiae GPI-anchored protein gp115 in morphogenesis and cell separation. J Bacteriol 175, 1879-1885.

Porta, A., Ramon, A. M. \& Fonzi, W. A. (1999). PRR1, a homolog of Aspergillus nidulans palF, controls pH-dependent gene expression and filamentation in Candida albicans. J Bacteriol 181, 7516-7523.

Proctor, R. H., Desjardins, A. E., Plattner, R. D. \& Hohn, T. M. (1999). A polyketide synthase gene required for biosynthesis of fumonisin mycotoxins in Gibberella fujikuroi mating population A. Fungal Genet Biol 27, 100-112.

Proctor, R. H., Brown, D. W., Plattner, R. D. \& Desjardins, A. E. (2003). Co-expression of 15 contiguous genes delineates a fumonisin biosynthetic gene cluster in Gibberella moniliformis. Fungal Genet Biol 38, 237-249.

Ram, A. F. J. \& Klis, F. M. (2006). Identification of fungal cell wall mutants using susceptibility assays based on Calcofluor white and Congo red. Nat Protoc 1, 2253-2256.

Ram, A. F., Wolters, A., Ten Hoopen, R. \& Klis, F. M. (1994). A new approach for isolating cell wall mutants in Saccharomyces cerevisiae by screening for hypersensitivity to calcofluor white. Yeast 10, 1019-1030.

Roncero, C., Valdivieso, M. H., Ribas, J. C. \& Duran, A. (1988). Isolation and characterization of Saccharomyces cerevisiae mutants resistant to calcofluor white. J Bacteriol 170, 1950-1954.

Sagaram, U. S., Kolomiets, M. \& Shim, W. B. (2006). Regulation of fumonisin biosynthesis in Fusarium verticillioides-maize system. Plant Pathol J 22, 203-210.

Sambrook, J. \& Russell, D. W. (2001). Molecular Cloning: a Laboratory Manual, 3rd edn. Cold Spring Harbor, NY: Cold Spring Harbor Laboratory. 
Saporito-Irwin, S. M., Birse, C. E., Sypherd, P. S. \& Fonzi, W. A. (1995). PHR1, a pH-regulated gene of Candida albicans, is required for morphogenesis. Mol Cell Biol 15, 601-613.

Shaw, B. D. \& Hoch, H. C. (1999). The pycnidiospore of Phyllosticta ampelicida: surface properties involved in substratum attachment and germination. Mycol Res 103, 915-924.

Shim, W. B. \& Woloshuk, C. P. (1999). Nitrogen repression of fumonisin B1 biosynthesis in Gibberella fujikuroi. FEMS Microbiol Lett 177, 109-116.

Shim, W. B. \& Woloshuk, C. P. (2001). Regulation of fumonisin B1 biosynthesis and conidiation in Fusarium verticillioides by a cyclin-like (C-type) gene, FCC1. Appl Environ Microbiol 67, 1607-1612.

Shim, W. B., Sagaram, U. S., Choi, Y. E., So, J., Wilkinson, H. H. \& Lee, Y. W. (2006). FSR1 is essential for virulence and female fertility in Fusarium verticillioides and F. graminearum. Mol Plant Microbe Interact 19, 725-733.

Smith, E. E. \& Goldstein, I. J. (1967). Protein-carbohydrate interaction. V. Further inhibition studies directed toward defining the stererochemical requirements of the reactive sites of concanavalin A. Arch Biochem Biophys 121, 88-95.

Tilburn, J., Sarkar, S., Widdick, D. A., Espeso, E. A., Orejas, M., Mungroo, J., Penalva, M. A. \& Arst, H. N. (1995). The Aspergillus Pacc zinc-finger transcription factor mediates regulation of both acidexpressed and alkaline-expressed genes by ambient $\mathrm{pH}$. EMBO J 14, 779-790.

Upadhyay, S. \& Shaw, B. D. (2006). A phosphoglucose isomerase mutant in Aspergillus nidulans is defective in hyphal polarity and conidiation. Fungal Genet Biol 43, 739-751.

White, D. G. (1999). Compendium of Corn Disease, 3rd edn. St Paul, MN: APS Press.

Yu, J. H., Hamari, Z., Han, K. H., Seo, J. A., Reyes-Dominguez, Y. \& Scazzocchio, C. (2004). Double-joint PCR: a PCR-based molecular tool for gene manipulations in filamentous fungi. Fungal Genet Biol 41, 973-981.

Edited by: J.-R. Xu 